Modelización CFD para determinar el comportamiento del fluido en tuberías de PVC

\title{
Modelización CFD para determinar el comportamiento del fluido en tuberías de PVC
}

\section{CFD modeling to determine the behavior of the fluid in PVC pipes}

\section{Modelagem CFD para determinar o comportamento do fluido em tubos de PVC}

\author{
Marco A. Ordoñez-Viñán ${ }^{\mathrm{I}}$ \\ marco.ordonez@espoch.edu.ec \\ Sócrates M. Aquino-Arroba II \\ saquino@espoch.edu.ec \\ Lenin S. Orozco-Cantos III \\ 1sororzco@espoch.edu.ec \\ Edwin R. Pozo-Safla IV \\ edwin.pozo@espoch.edu.ec \\ Edwin Á. Jácome-Domínguez ${ }^{\mathrm{V}}$ \\ edwin.jacome@espoch.edu.ec
}

Recibido: 06 de agosto de 2017 * Corregido: 11 de octubre de 2017 * Aceptado: 21 de noviembre de 2017

I. Ingeniero Mecánico, Docente de la Escuela Politécnica de Chimborazo, Riobamba, Chimborazo, Ecuador.

II. Magister en Diseño Producción y Automatización Industrial, Ingeniero Mecánico, Docente de la Escuela Politécnica de Chimborazo, Riobamba, Chimborazo, Ecuador.

III. Magister en Eficiencia Energética, Ingeniero Mecánico, Docente de la Escuela Politécnica de Chimborazo, Riobamba, Chimborazo, Ecuador.

Iv. Magister en Diseño Producción y Automatización Industrial, Ingeniero Mecánico, Docente de la Escuela Politécnica de Chimborazo, Riobamba, Chimborazo, Ecuador.

v. Magister en Eficiencia Energética, Ingeniero Mecánico, Docente de la Escuela Politécnica de Chimborazo, Riobamba, Chimborazo, Ecuador. 


\title{
Resumen
}

El objetivo principal en este estudio es desarrollar un modelo CFD (Computational Fluid Dynamics) para el análisis y simulación de los perfiles de velocidad en el banco de pérdidas del laboratorio de Turbomaquinaria Hidráulica. El perfil de velocidad es la simulación del comportamiento del fluido interno en el banco de tuberías de PVC. El modelo desarrollado para la simulación de los perfiles de velocidad se fundamenta en las ecuaciones de Navier -Stokes de flujo incompresible, que tienen el principio de la conservación de masa y cantidad de movimiento. Para la solución de estas ecuaciones se utilizó ANSYS CFX basado en tres etapas de simulación por ordenador; Pre-procesamiento, procesamiento y post-procesamiento. El pre-proceso comprende el estudio del modelo físico y las variables que intervienen con la finalidad de una simulación lo más próxima a la realidad. En la etapa del procesamiento se generan resultados en estado estacionario el flujo del fluido. La validación de resultados, se realizó con el cálculo analítico del perfil de velocidades mediante los datos adquiridos en el banco de pruebas y con los resultados obtenidos en la simulación mediante ANSYS CFX, con un criterio del juicio de la ingeniería utilizando el mínimo porcentaje de error.

Palabras clave: modelación; fluidos; tuberías.

\begin{abstract}
The main objective in this study is to develop a CFD model (Computational Fluid Dynamics) for the analysis and simulation of the velocity profiles in the Loss Bank of the Hydraulic Turbomachinery laboratory. The velocity profile is the simulation of the behavior of the internal fluid in the PVC pipe bank. The model developed for the simulation of velocity profiles is based on Navier's equations Stokes of incompressible flow, which have the principle of conservation of mass and momentum. For the solution of these equations ANSYS CFX was used based on three stages of computer simulation; Pre-processing, processing and post-processing. The pre-process includes the study of the physical model and the variables that intervene with the purpose of a simulation that is closest to reality. In the stage of the processing results in steady state the flow of the fluid are generated. The validation of results was carried out with the analytical calculation of the velocity profile by means of the data acquired in the test bench and with the results obtained in the simulation using ANSYS CFX, with an engineering judgment criterion using the minimum error percentage.
\end{abstract}

Keywords: modeling; fluids; pipelines. 


\section{Resumo}

O principal objetivo deste estudo é desenvolver um modelo CFD (Computational Fluid Dynamics) para análise e simulação de perfis de velocidade no banco de perdas do laboratório Turbomaquinaria Hidráulica. O perfil de velocidade é a simulação do comportamento do fluido interno no banco de tubos de PVC. O modelo desenvolvido para a simulação dos perfis de velocidade baseia-se nas equações de Navier. As sondas de fluxo incompressível, que têm o princípio de conservação de massa e momentum. Para a solução dessas equações, o ANSYS CFX foi utilizado com base em três estágios de simulação computacional; Pré-processamento, processamento e pós-processamento. O préprocesso inclui o estudo do modelo físico e as variáveis que intervêm com o objetivo de uma simulação mais próxima da realidade. $\mathrm{Na}$ fase dos resultados do processamento em estado estacionário, o fluxo do fluido é gerado. A validação dos resultados foi realizada com o cálculo analítico do perfil de velocidade por meio dos dados adquiridos no banco de teste e com os resultados obtidos na simulação usando ANSYS CFX, com um critério de julgamento de engenharia usando a porcentagem mínima de erro.

Palavras chave: modelagem; fluidos; tubos.

\section{Introducción}

La dinámica de fluidos computacional (CFD) ha sido desarrollada con la finalidad de dar soluciones a problemas complejos de la mecánica de fluidos en busca de predecir el flujo de fluidos, transferencia de calor y masa, reacciones químicas, etc. Para la solución de dichos problemas se utilizan métodos numéricos con vistas a resolver los modelos matemáticos que gobiernan el fenómeno, como son conservación de masa, momento, energía, cantidad de momento.

El paso de un fluido por una tubería, consiste en flujos que quedan completamente limitados por superficies sólidas como en sistemas de calefacción, enfriamiento y en redes de distribución de fluidos. En las tuberías, generalmente se tienen dos tipos de flujos laminar y turbulento, dependiendo de la velocidad del fluido y el diámetro de las tuberías por donde fluye. En la práctica, es más frecuente un flujo turbulento. La naturaleza del flujo a través de un tubo está determinada por el valor que tome el número de Reynolds siendo este un número adimensional dependiente de la densidad, viscosidad, velocidad de flujo y el diámetro de la tubería. 
En esta investigación se centra en el análisis del comportamiento del fluido en el banco de tuberías, donde el conducto está totalmente lleno con el fluido y el flujo se impulsa principalmente mediante una bomba en un ciclo cerrado.

\section{Metodología}

La dinámica de fluidos computacional basado en el método de volúmenes finitos parte de la división de un volumen de fluido en un número finito de volúmenes de control como se muestra en la figura 1.

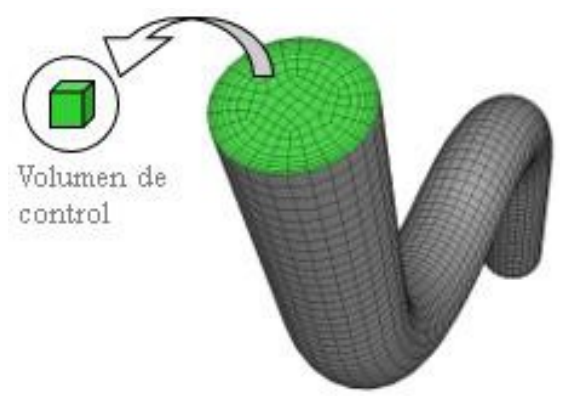

Figura 1. Discretización de una región de fluido en volúmenes finitos de control.

El software CFD obtiene la solución de los problemas de Mecánica de Fluidos resolviendo de forma numérica las ecuaciones de Navier-Stokes, de flujo turbulento incompresible estacionario.

La ecuación de conservación de masa para flujo estacionario de un fluido viscoso, newtoniano e incompresible, las ecuaciones de movimiento son las ecuaciones de continuidad. $\vec{\nabla} \cdot \vec{V}=0$

La ecuación de Navier-Stokes es una ecuación de transporte que representa la cantidad de movimiento lineal en el dominio computacional.

A continuación se representa e identifica cada elemento de la ecuación gobernante.

$$
\begin{gathered}
\vec{a} \rightarrow \vec{A} \quad \vec{a} \quad \overrightarrow{2} \\
{[\nabla \cdot V] V=-\frac{\vec{\rho}}{\nabla} P^{\prime}+v \nabla V}
\end{gathered}
$$

En el análisis por medio de CFD debe seguir la secuencia que se muestra en la figura 2. 
Modelización CFD para determinar el comportamiento del fluido en tuberías de PVC

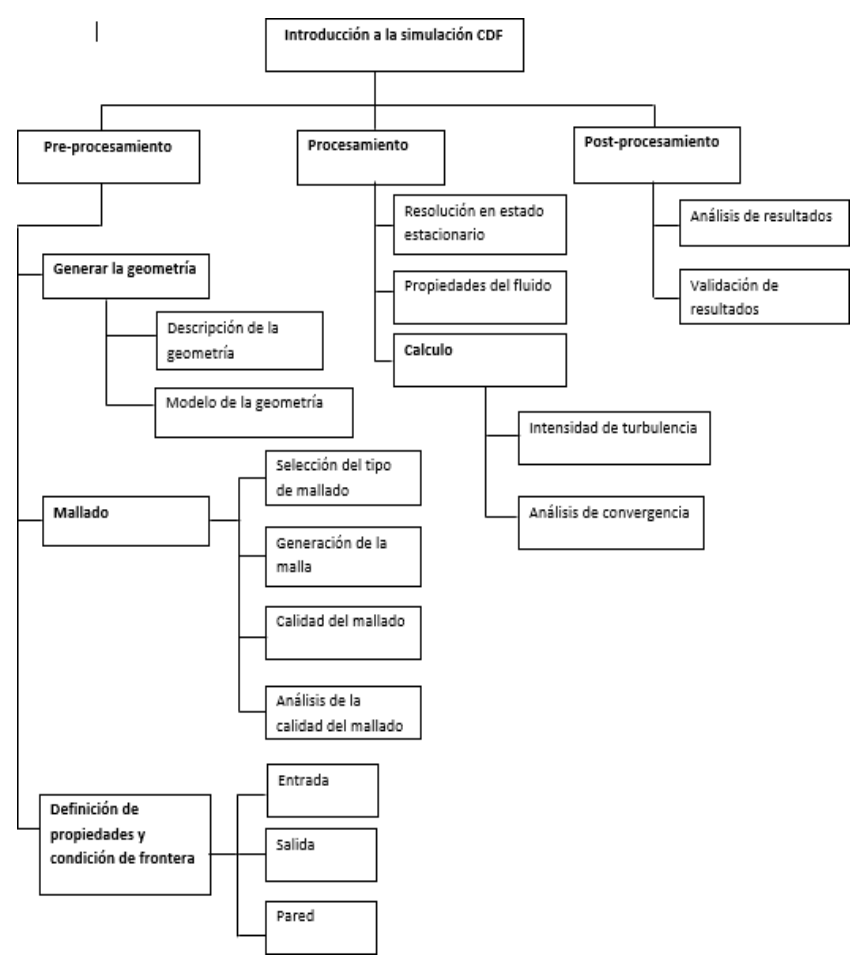

Figura 2. Diagrama metodológico para CFD

La etapa de pre-procesamiento es la que define las características generales del problema de simulación y requiere un entendimiento y control adecuado del fenómeno de tal modo que se garanticen los resultados verídicos. Para la cual se debe seguir los siguientes pasos:

- Creación de un modelo CAD del dominio computacional que represente de manera física la situación experimental.

En la figura 3 se muestra en la tubería por donde debe circular el volumen de fluido que se requiere estudiar.

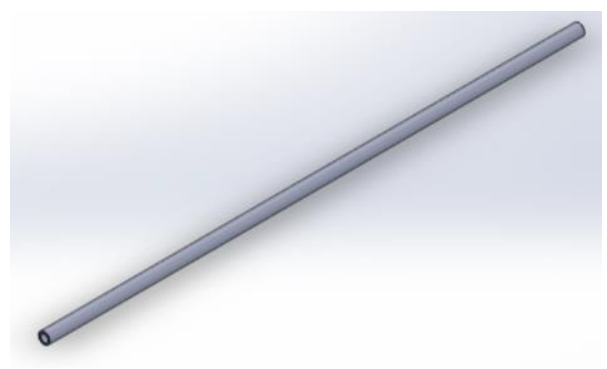

Figura 3. Modelo Geométrico 
Generación de la malla que se ajusta al modelo CAD según sea el caso estudiado se definen los tipos de mallado que conforman la geometría.

El objetivo de un código de CFD es la resolución de las ecuaciones de Navier-Stokes para un flujo con unas características determinadas y para una geometría concreta. El código de CFD resuelve dichas ecuaciones en una serie de puntos de la geometría en cuestión mediante métodos numéricos que transforman las ecuaciones diferenciales en ecuaciones algebraicas, proceso que se denomina discretización espacial a emplear depende del tipo de discretización, el primer paso que se da en resolver las ecuaciones es la selección de los puntos en los que se llevara a cabo la discretización, proceso que se denomina generación del mallado. En la figura 4 se muestra la forma de la malla generada para el análisis del fenómeno en estudio.

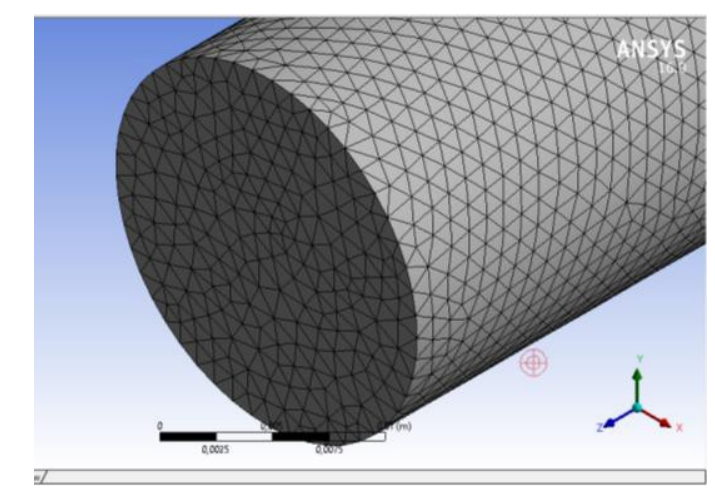

Details of "Mesh"
\begin{tabular}{|l|l|}
\hline Mesh Morphing & Disabled \\
\hline Defeaturing & \\
\hline Pinch Tolerance & Default $(0,448320 \mathrm{~mm})$ \\
\hline Generate Pinch on Refresh & No \\
\hline Automatic Mesh Based Defeaturing & On \\
\hline$\square$ Defeaturing Tolerance & Default $(0,249070 \mathrm{~mm})$ \\
\hline Statistics & \\
\hline$\square$ Nodes & 1114599 \\
\hline$\square$ Elements & 5902819 \\
\hline
\end{tabular}

Figura 4. Mallado del volumen de fluido

Para proceder a construir el mallado de una geometría, el primer paso es seleccionar el tipo o procedimiento de mallado. En el ANSYS-CFX el generador de mallas tiene varios métodos disponibles para mallado de volúmenes. Los métodos disponibles en ANSYS-CFX tienen ciertas ventajas sobre otro al emplearse en ciertos casos específicos. Por eso se debe seleccionar el método adecuado para realizar el mallado de las tuberías, donde se debe tener en cuenta la geometría para 
seleccionar el método que menos deforme el dominio y aquel que represente de la mejor manera el caso a modelar. Por lo tanto es muy importante la evaluación de la calidad de malla, ANSYS posee varios criterios matemáticos que permite ver la calidad de malla de un elemento, para el estudio se usa la condición de oblicuidad. Una vez revisado las características de todos los métodos, se llega a determinar que una buena opción a emplearse es el mallado tetraédrico (tetraedros). En figura 5 se muestra la evaluación de la calidad de malla para el volumen de fluido mientras que la tabla 1 indica los valores recomendados que la malla debe cumplir para un análisis adecuado.

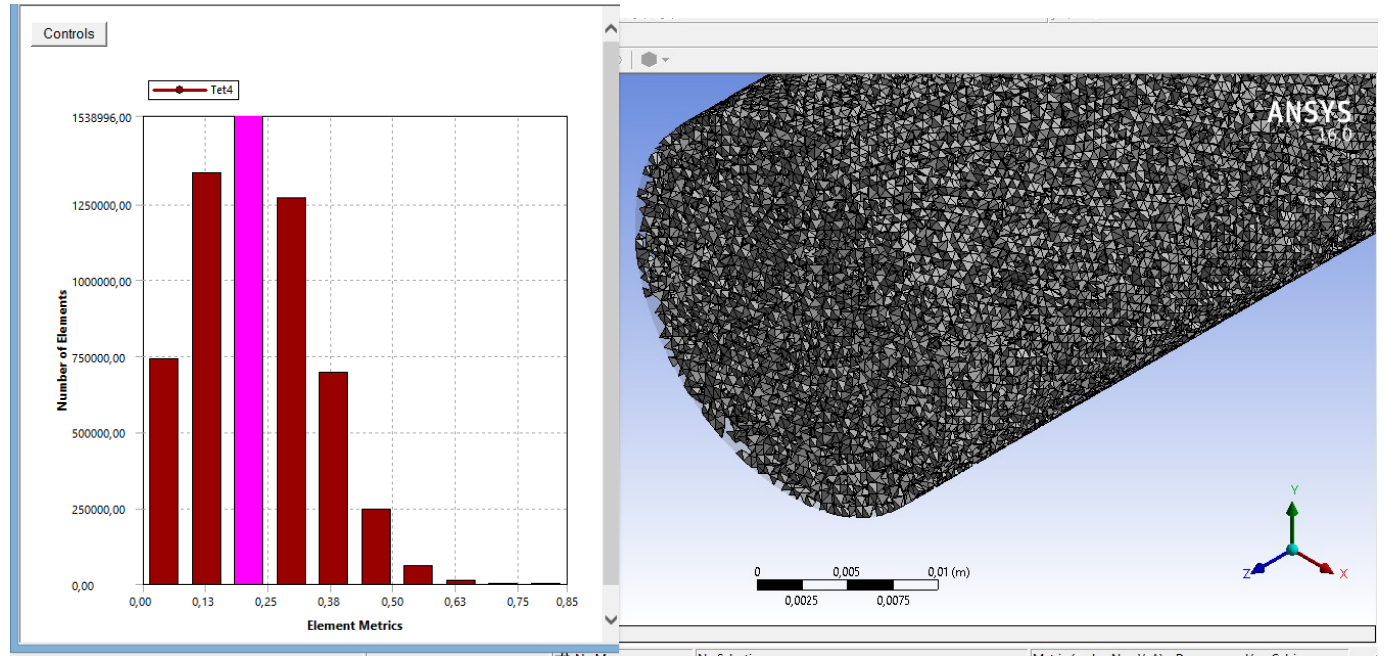

Figura 5. Evaluación de calidad de malla

Tabla 1: Valores recomendados para evaluar la calidad de malla

\begin{tabular}{|l|l|}
\hline Valores de oblicuidad & Indicadores de calidad \\
\hline 1 & Degenerado \\
\hline $0,90-<1$ & Muy malo \\
\hline $0,75-0,90$ & Pobre \\
\hline $0,50-0,75$ & Regular \\
\hline $0,25-0,50$ & Bueno \\
\hline$>0-0,25$ & Excelente \\
\hline 0 & Equilátero perfecto \\
\hline
\end{tabular}

- Definición física del modelo, que componen la simulación, las condiciones de frontera y los parámetros de solución. 
La figura 6 muestra las condiciones de frontera del volumen de fluido en estudio.
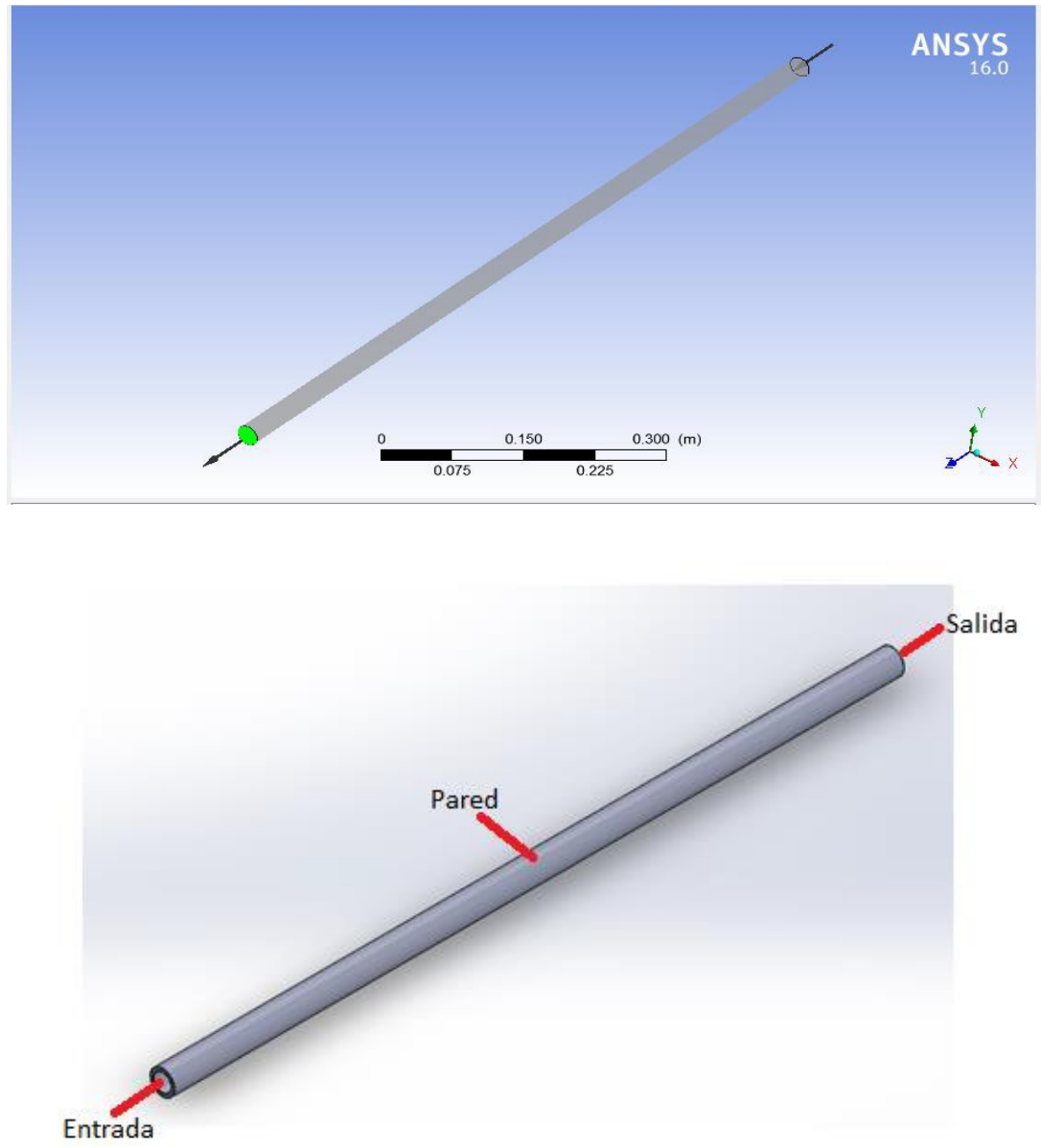

Figura 6. Condiciones de frontera del modelo

\section{Resultados}

Los resultados de solución en estado estable en CFD es el análisis de los perfiles. En la simulación solo se analizan los perfiles de velocidad de flujo turbulento, este tipo de flujo es más evidente en las prácticas reales.

El flujo turbulento a lo largo de una pared se puede considerar con cuatro regiones, que se caracterizan por la distancia desde la pared. La capa delgada junto a la pared llamada subcapa viscosa (o laminar o lineal o de pared). El perfil de velocidad en esta capa es casi lineal, y el flujo es de líneas de corriente aproximadamente paralelas como en el flujo laminar. (CIMBALA, 2006) 
Junto a la subcapa viscosa está la capa de amortiguamiento, en la que los efectos turbulentos se vuelven significativos, pero el flujo todavía es dominado por los efectos viscosos. Sobre la capa de amortiguamiento está la capa de traslape (o transición), también llamada subcapa inercial, en la que los efectos turbulentos son mucho más significativos, pero todavía sin dominar. (CIMBALA, 2006)

Sobre esta capa se encuentra la capa exterior o turbulenta en la parte restante del flujo en la que los efectos turbulentos dominan sobre los efectos de difusión molecular.

Se determina el cálculo analítico de los perfiles de velocidad, para la respectiva validación de resultados con los perfiles de velocidades obtenidos en la simulación del software ANSYS-CFX. La distribución de las velocidades locales en el interior de la tubería, estas velocidades locales define la forma del perfil turbulento con su respectivo número de Reynolds, en el área transversal de la tubería, considerando la velocidad en la pared igual a cero y se va incrementando hasta alcanzar una velocidad máxima en el eje central de la tubería.

En la figura 7, 8 y 9 se determinan el comportamiento del fluido en el interior de las tuberías, donde se identifica en cada apertura con su respectivo número de Reynolds el perfil de velocidad turbulento en régimen estacionario.

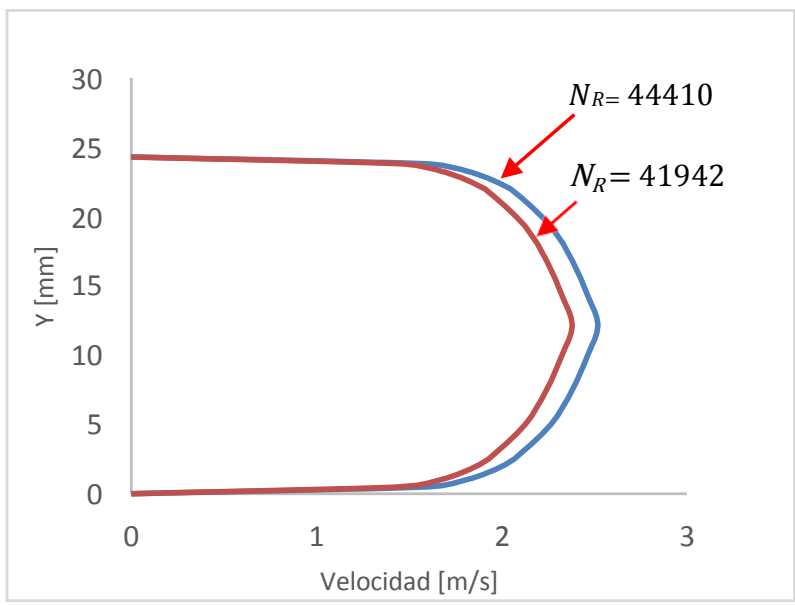

Figura 7. Perfil de velocidad en la tubería de 1 plg 
Modelización CFD para determinar el comportamiento del fluido en tuberías de PVC

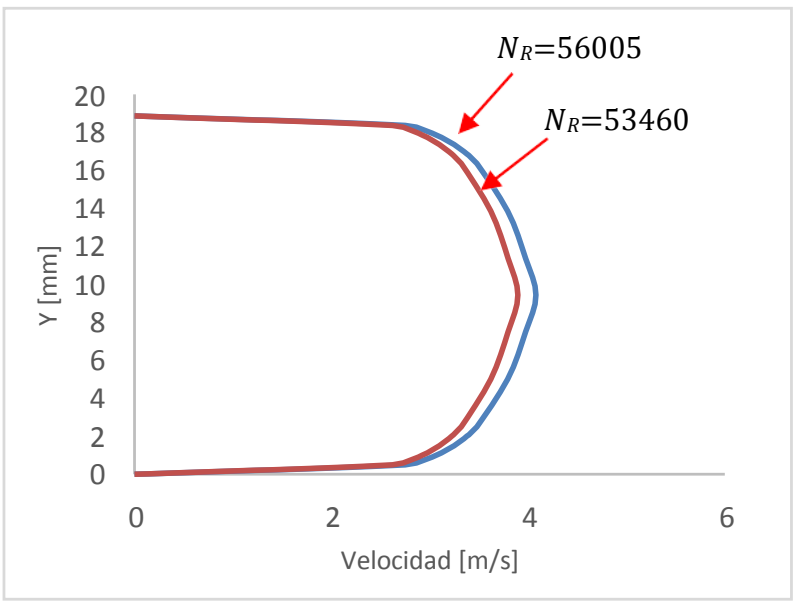

Figura 8. Perfil de velocidad en la tubería $3 / 4 \mathrm{plg}$

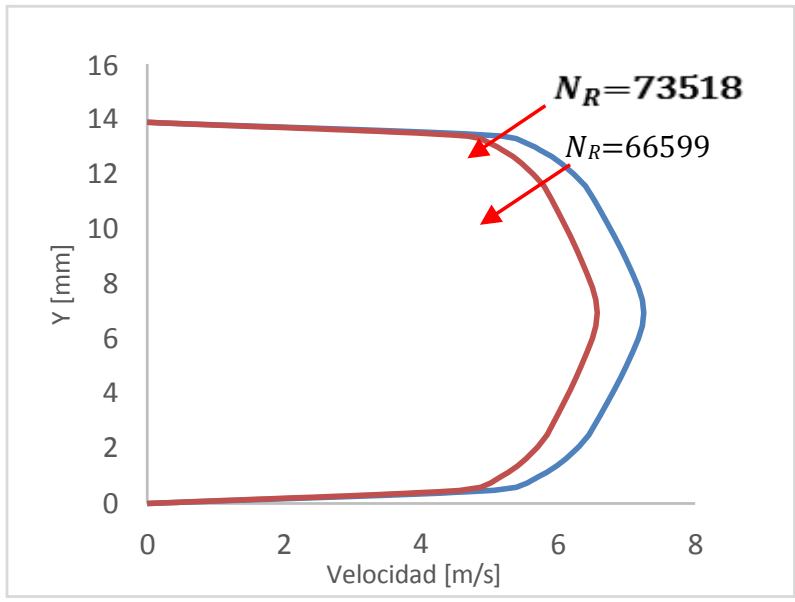

Figura 9. Perfil de velocidad en la tubería $1 / 2 \mathrm{plg}$

En la simulación realizada del fenómeno físico a través del modelo matemático desarrollado mediante la CFD es muy importante analizar los resultados.

Para la validación de resultados se fundamentó en la forma del perfil de velocidad turbulento mediante el análisis de ANSYS-CFX y las prácticas experimentales realizadas en el banco de pérdidas. 
Tabla 2. Análisis de resultados

\begin{tabular}{|l|l|l|l|l|}
\hline $\begin{array}{l}\text { Apertura } \\
\text { [vueltas] }\end{array}$ & $\mathbf{d}_{\text {int }}$ & $\begin{array}{l}\text { Resultado } \\
\text { ANSYS- } \\
\text { CFX }\end{array}$ & $\begin{array}{l}\text { Resultado } \\
\text { experimental }\end{array}$ & $\begin{array}{l}\text { \% } \\
\text { Error } \\
\text { velocidad } \\
\text { máxima } \\
{[\mathbf{m} / \mathbf{s}]}\end{array}$ \\
\hline 7 & $\begin{array}{l}\text { velocidad } \\
\text { máxima } \\
{[\mathbf{m} / \mathbf{s}]}\end{array}$ & \\
\hline 1 & 24,30 & 2,63 & 2,52 & 4.36 \\
\hline 7 & 24,30 & 2,37 & 2,38 & 0,42 \\
\hline 1 & 18,84 & 3,87 & 4,08 & 6.40 \\
\hline 7 & 18,84 & 3,49 & 3,82 & 8,60 \\
\hline 1 & 13,86 & 7,65 & 7,24 & 5,66 \\
\hline & 13,86 & 6,42 & 6,57 & 2,28 \\
\hline
\end{tabular}

En la tabla 2 se indican los valores de las velocidades máximas que forman el perfil de velocidad en el centro de las tuberías en cada apertura de la válvula de compuerta. Los resultados obtenidos de la simulación en el Software ANSYS-CFX, predicen de manera muy próxima el comportamiento real del fenómeno.

Para la estimación del porcentaje de error de las simulaciones en ANSYS-CFX se considera el aspecto más importante a predecir, es decir la velocidad máxima de los perfiles de velocidad. El porcentaje de error varía desde un valor de $0,42 \%$ a 8,6 \% en los valores de las velocidades máximas que forman los perfiles de velocidad.

El porcentaje de error más elevado en la simulación CFD es en el tramo de tubería de tres cuartos de pulgada con una diferencia de $0,33 \mathrm{~m} / \mathrm{s}$ de la velocidad máxima que forma el perfil de velocidad en el eje central de la tubería en la apertura de cierre de una vuelta con la válvula de compuerta.

\section{Conclusiones}

Se cumplió el objetivo de la simulación del flujo incompresible estacionario en tuberías de PVC del banco de pérdidas del laboratorio de Turbomaquinaria mediante la CFD prediciendo una simulación real. 
Modelización CFD para determinar el comportamiento del fluido en tuberías de PVC

Con los resultados obtenidos en el análisis experimental y la simulación numérica CFD mediante el software ANSYS-CFX se concluye que el modelo CFD simula de una manera correcta a la realidad en el análisis de los perfiles de velocidad.

En la simulación CFD, analizamos solo flujo de régimen turbulento donde se determinan el comportamiento del fluido en diferentes capas en el interior de la tubería.

El mallado tetraédrico es el mejor elemento para simular flujo turbulento. Al validar los resultados del análisis de los perfiles de velocidad en pérdidas primarias en tuberías de PVC. Se determina que el máximo error obtenido es de 8.6\% considerando un valor aceptable para simulación estacionario.

En el banco de pérdidas, se concluye que los instrumentos están calibrados debidamente para realizar las prácticas.

\section{Referencias bibliográficas}

ANSYS.INC. 2015. ANSYS. [En línea] Diciembre de 2015. [Citado el: 10 de marzo de 2017.] http://www.ansys.com/Products/Simulation+Technology/Fluid+Dynamics/Fluid+Dynamics+Produc ts/ANSYS+CFX.

BAKKER, Andre. 2012. The Colorful Fluid Mixing Gallery. [En línea] 2012. http://www.bakker.org/. CIMBALA, YUNUS A. CENGEL JOHN M. 2006. Mecánica de Fluidos. México: McGraw-Hill, 2006.

FARIAS, Ernesto. 2015. Monografias.com S.A. [En línea] 2015. [Citado el: 31 de abril de 2017.] http://www.monografias.com/trabajos102/empleo-metodos-numericos-mejoramiento-rotoresturbinas-eolicas/image003.jpg.

JOHN D. ANDERSON JR. 1995. Computational Fluid Dynamics. United States of America : McGraw-Hill, 1995.

MOTT, ROBERT L. 1996. Mecánica De Fluidos Aplicada. México: PEARSON, 1996.

OMEGA Engineering. 2015. OMEGA.com. [En línea $] 2017$. http://mx.omega.com/pptst/PX302.html. 
Modelización CFD para determinar el comportamiento del fluido en tuberías de PVC

PASOR, ANTONIO BARRERO RIPOLL- MIGUEL PEREZ- SABORID SANCHEZ-. 2005.

Fundamentos y Aplicaciones de la Mecanica de Fluidos. España: McGraw-Hill, 2005.

PODGORNIK, Rudolf. 2007. Department of Theoretical Physics (F-1). [En línea] Marzo de 2007.

[Citado el: 15 de abril de 2015.] http://www-f1.ijs.si/ rudi/sola/Turbulence-models-in-CFD.pdf.

ROBERSON, CLAYTON T. CROWE DONALD F. ELGER JOHN A. 2002. Mecánica de Fluidos. Mexico : CECSA, 2002.

Temperature Instrumentation. 2015. INSTRUMART. [En línea $] 2017$. https://www.instrumart.com/assets/ADT914-datasheet.pdf.

Voller, Vaughan R. 2009. Basic Control Volume Finite Element Methods for Fluids and Solids. USA: World Scientific Publishing Co. Pte. Ltd., 2009.

WHITE, FRANK M. 2012. MECANICA DE FLUIDOS. Mexico: McGraw-Hill, 2012.

WordPress. 2014. Barba de Kutta. [En línea] 2014. [Citado el: 21 de Marzo de 2017.] https://porlasbarbasdekutta.files.wordpress.com/2013/01/captura-de-pantalla-2013-01-05-a-las-1402-48.png. 